\title{
Schmidt, Christian
}

\section{Demografischer Wandel und Entwicklung berufsbildender Schulen}

Faßhauer, Uwe [Hrsg.]; Fürstenau, Bärbel [Hrsg.]; Wuttke, Eveline [Hrsg.]: Grundlagenforschung zum Dualen System und Kompetenzentwicklung in der Lehrerbildung. Opladen ; Berlin ; Farmington Hills, Mich. : Verlag Barbara Budrich 2011, S. 143-152. - (Schriftenreihe der Sektion Berufs- und Wirtschaftspädagogik der Deutschen Gesellschaft für Erziehungswissenschaft (DGfE))

Quellenangabe/ Reference:

Schmidt, Christian: Demografischer Wandel und Entwicklung berufsbildender Schulen - In: Faßhauer, Uwe [Hrsg.]; Fürstenau, Bärbel [Hrsg.]; Wuttke, Eveline [Hrsg.]: Grundlagenforschung zum Dualen System und Kompetenzentwicklung in der Lehrerbildung. Opladen ; Berlin ; Farmington Hills, Mich. : Verlag Barbara Budrich 2011, S. 143-152 - URN: urn:nbn:de:0111-opus-70748 - DOI: 10.25656/01:7074

https://nbn-resolving.org/urn:nbn:de:0111-opus-70748

https://doi.org/10.25656/01:7074

in Kooperation mit / in cooperation with:

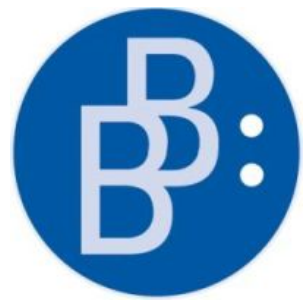

https://www.budrich.de

\section{Nutzungsbedingungen}

Gewährt wird ein nicht exklusives, nicht übertragbares, persönliches und beschränktes Recht auf Nutzung dieses Dokuments. Dieses Dokument ist ausschließlich für den persönlichen, nicht-kommerziellen Gebrauch bestimmt. Die Nutzung stellt keine Übertragung des Eigentumsrechts an diesem Dokument dar und gilt vorbehaltlich der folgenden Einschränkungen Auf sämtlichen Kopien dieses Dokuments müssen alle Urheberrechtshinweise und sonstigen Hinweise auf gesetzlichen Schutz beibehalten werden. Sie dürfen dieses Dokument nicht in irgendeiner Weise abändern, noch dürfen Sie dieses Dokument für öffentliche oder kommerzielle Zwecke vervielfältigen, öffentlich ausstellen, aufführen, vertreiben oder anderweitig nutzen.

Mit der Verwendung dieses Dokuments erkennen Sie die Nutzungsbedingungen an.

\section{Terms of use}

We grant a non-exclusive, non-transferable, individual and limited right to using this document.

This document is solely intended for your personal, non-commercial use. Use of this document does not include any transfer of property rights and it is conditional to the following limitations: All of the copies of this documents must retain all copyright information and other information regarding legal protection. You are not allowed to alter this document in any way, to copy it for public or commercial purposes, to exhibit the document in public, to perform, distribute or otherwise use the document in public.

By using this particular document, you accept the above-stated conditions of use.

\section{Kontakt / Contact:}

\section{peDOCS}

DIPF | Leibniz-Institut für Bildungsforschung und Bildungsinformation Informationszentrum (IZ) Bildung

E-Mail: pedocs@dipf.de

Internet: www.pedocs.de

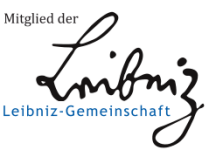


Grundlagenforschung zum Dualen System und Kompetenzentwicklung in der Lehrerbildung 


\section{Uwe Faßhauer}

Bärbel Fürstenau

Eveline Wuttke (Hrsg.)

\section{Grundlagenforschung zum Dualen System und Kompetenzentwicklung in der Lehrerbildung}

Verlag Barbara Budrich Opladen • Berlin • Farmington Hills, MI 2011 
Bibliografische Information der Deutschen Nationalbibliothek

Die Deutsche Nationalbibliothek verzeichnet diese Publikation in der Deutschen

Nationalbibliografie; detaillierte bibliografische Daten sind im Internet über

http://dnb.d-nb.de abrufbar.

(C) Dieses Werk ist im Verlag Barbara Budrich erschienen und steht unter folgender Creative Commons Lizenz: http://creativecommons.org/licenses/by-nc-nd/3.0/de/ Verbreitung, Speicherung und Vervielfältigung erlaubt, kommerzielle Nutzung und Veränderung nur mit Genehmigung des Verlags Barbara Budrich.

\footnotetext{
SOMERIGHTS RESERVED
}

Dieses Buch steht im OpenAccess Bereich der Verlagsseite zum kostenlosen Download bereit (http://dx.doi.org/10.3224/86649461)

Eine kostenpflichtige Druckversion (Printing on Demand) kann über den Verlag bezogen werden. Die Seitenzahlen in der Druck- und Onlineversion sind identisch.

\section{ISBN 978-3-86649-461-9 \\ DOI $\quad 10.3224 / 86649461$}

Umschlaggestaltung: Umschlaggestaltung: bettina lehfeldt graphic design, Kleinmachnow

Verlag Barbara Budrich, http://www.budrich-verlag.de 
Inhaltsverzeichnis

Vorwort.............................................. 7

\section{Teil I: Kompetenzentwicklung in der Lehrerbildung für berufliche Schulen}

Cindy Grzanna

Die Subjektiven Theorien von Absolventen der

Wirtschaftspädagogik über ihre Berufsidentität - Ergebnisse einer explorativen Studie.

Doreen Holtsch

Fachdidaktische Kompetenz (künftiger) Lehrender im

kaufmännischen Bereich

Mareike Junghanns

Die empirische Evidenz der Handlungsfelder von LehrerInnen in den KMK-Empfehlungen zu den Bildungs- und Fachwissenschaften

Ulrike Weyland/ Eveline Wittmann

Zur Einführung von Praxissemestern: Bestandsaufnahme, Zielsetzungen und Rahmenbedingungen.

Volkmar Herkner/Jörg-Peter Pahl

Berufliche Fachrichtungen - Pragmatik, Probleme und Perspektiven............................................... 


\section{Teil II: Grundlagenforschung zum Dualen System}

Stephan Schumann/ Franz Eberle

Bedeutung und Verwendung schwierigkeitsbestimmender Aufgabenmerkmale für die Erfassung ökonomischer und beruflicher Kompetenzen.............................. 77

Daniel Pittich

Studie zur Überprüfung des Zusammenhangs von Verständnis und Fachkompetenz bei Auszubildenden des Handwerks.

Frank Musekamp

Validierung eines Multiple-Choice-Instruments zur Erfassung von Kompetenzen in der Domäne Kfz-Service \& Mandy Hommel

Aufmerksamkeitsverlauf - Fremdbeobachtung und

Eigeneinschätzung.

Raymond Djaloeis/Martin Frenz/Simon Heinen/

Christopher M. Schlick

Diagnose von Energieberatungskompetenz....

Christian Schmidt

Demografischer Wandel und Entwicklung berufsbildender

Schulen

Karin Wirth

Verknüpfung schulischer und betrieblicher Ausbildungsanteile in konsekutiven Ausbildungsformen.... 


\title{
Demografischer Wandel und Entwicklung berufsbildender Schulen
}

\author{
Christian Schmidt
}

\section{Problemstellung}

Der demografische Wandel stellt das berufliche Bildungssystem auf zweierlei Weise vor neue Herausforderungen. Auf der einen Seite wird er aller Voraussicht nach einen Mangel an Fachkräften nach sich ziehen und das berufliche Bildungssystem wird dem Anspruch begegnen müssen, dieser Problematik entgegen zu wirken. Auf der andere Seite bedeutet der demografische Wandel für die berufsbildenden Schulen einen Rückgang der Schülerzahlen in den unterschiedlichen Schulformen, von der dualen Ausbildung über vollqualifizierende vollschulische Ausbildungsgänge bis hin zu den Schulformen des Übergangssystems und jene, die zu einem Hochschulzugang führen. Hier besteht die Herausforderung darin, die Versorgung mit Schulstandorten sicherzustellen und den Jugendlichen regional Bildungsmöglichkeiten offenzuhalten. Aktuell wird der Aspekt der Fachkräfteproblematik und den Auswirkungen auf das Berufsbildungssystem stark diskutiert, die Entwicklungsprobleme berufsbildender Schulen treten dagegen in den Hintergrund. Dieser Widerspruch sollte aufgelöst werden zugunsten einer Betrachtungsweise über die Auswirkungen des demografischen Wandels auf das Berufsbildungssystem, welche die Risiken für den Lernort berufsbildende Schule mit einbezieht.

Im Folgenden soll der Frage nachgegangen werden, inwieweit die Herausforderungen für die berufsbildenden Schulen durch den demografischen Wandel das Ziel beeinträchtigen, dem Fachkräftemangel positiv entgegenzuwirken. Dazu werden zunächst die Auswirkungen des demografischen Wandels auf die Schulabgangskohorten und die Prognosen zum erwarteten Fachkräftemangel dargestellt. Am Beispiel Nordrhein-Westfalens wird verdeutlicht, dass bereits jetzt strukturschwache Landkreise Rückgänge in den Schülerzahlen der Berufsschulen als Teil des dualen Systems erfahren. Abschließend werden Ansätze zur Entwicklung berufsbildender Schulen diskutiert, die es ermöglichen sollen, dass die Berufsschule als Teil des dualen Systems auf rückläufige Jahrgangsstärken zu reagieren in der Lage ist und so das duale System insgesamt einen Beitrag zur Linderung des Fachkräftemangels leisten kann. 


\section{Demografischer Wandel und Berufsbildungssystem}

Der demografische Wandel trifft das Berufsbildungssystem, wie alle anderen Bereiche des deutschen Bildungssystems auch, zunächst unmittelbar über den Rückgang der Kohorten in den für diesen Bildungsbereich wichtigen Altersklassen. Aufgrund der geringen Geburtenraten wird im Zeitraum von 2008 bis 2025 die Kohorte der 6- bis 19-jährigen um 24,3\% zurückgehen. Auch die Kohorte der 17- bis 25-jährigen wird in diesem Zeitraum um 15,4\% abnehmen (vgl. Euler 2010: 11). Insgesamt werden bis zum Jahr 2025 17\% weniger Bildungsteilnehmer in Westdeutschland und 14\% weniger Bildungsteilnehmer in Ostdeutschland erwartet (vgl. Autorengruppe Bildungsberichterstattung 2010: 154).

Den relativ sicheren Prognosen über die abnehmende Anzahl der Bildungsteilnehmer stehen solche über den zu erwartenden Fachkräftemangel gegenüber, die uneinheitlicher ausfallen. So sieht eine Prognose der Prognos AG einen Fachkräftemangel auf allen Qualifikationsebenen, also auch in dem für die berufliche Bildung einschlägigen „mittleren Qualifikationssegment“ herannahen, mit gewichtigen gesamtwirtschaftlichen Konsequenzen (vgl. Prognos AG 2010: 56 ff.). Skeptischer und differenzierter beurteilen Baethge, Laging und Philipps die Zukunft des Verhältnisses von Fachkräfteangebot und -nachfrage. Sie stützen sich auf die Projektionen der Bildungsberichterstattung und rechnen ab 2025 lediglich mit einem Gleichstand zwischen Angebot und Nachfrage unterhalb der Hochschulebene (vgl. Baethge/Laging/Philipps 2010: 3). Arbeitskraftengpässe sehen sie hier lediglich in den Gesundheits- und Sozialberufen, den Rechts- und Managementberufen, den künstlerischen und Medienberufen, den geistes- und sozialwissenschaftlichen Berufen sowie den Gastronomie- und Reinigungsberufen (vgl. Baethge/ Laging/Philipps 2010: 5). Diese Auswahl zeigt die für Dienstleistungsgesellschaften typische Kluft zwischen „good jobs“, welche einen Hochschulabschluss voraussetzen (Teile der Gesundheits- und Sozialberufe, Rechts und Managementberufe) sowie „bad jobs“, die geringe Qualifikationen mit geringem Gehalt und unsicherer Beschäftigung kombinieren (Gastronomie- und Reinigungsberufe) (vgl. Castells 2004: 234). Insgesamt kann davon ausgegangen werden, dass gerade die Abschätzung der Nachfrage nach qualifizierter Arbeit über einen Zeitraum von 15 Jahren mit Unsicherheiten behaftet sein muss, vor allem, wenn ein quasi „mechanisches“ Verhältnis zwischen Angebot und Nachfrage unterstellt wird.

Während die Gefahr nachlassender Schülerzahlen im Bereich der allgemeinbildenden Schulen als große, meist belastende Umwälzung eingeschätzt wird, die mit einem Austrocknen der Hauptschulen und der Schließung vieler Schulstandorte assoziiert wird (vgl. Autorengruppe Bildungsberichterstattung 2010: 172), ist es typisch für die berufliche Bildung, das auch positive Erwar- 
tungen an den demografischen Wandel gerichtet werden. So wird die Hoffnung formuliert, dass geringere Jahrgangsstärken zu einem Abschmelzen des Übergangssystems führen und wieder größere Anteile der von den allgemeinbildenden Schulen kommenden Schülerinnen und Schüler in vollqualifizierende Ausbildung übergehen könnten, da die Neuzugänge in das Übergangssystem aktuell rückläufig sind (vgl. Bosch 2010: 44; Autorengruppe Bildungsberichterstattung 2010: 97). Dieser Hoffnung liegt eine Verdrängungsthese zugrunde, wonach geringer qualifizierte Jugendliche in den Jahren des Ausbildungsplatzmangels durch von den Ausbildungsstellenanbietern bevorzugte höher qualifizierte Jugendliche verdrängt worden seien (vgl. Solga 2005: 102).

Zuletzt formulierte Euler hierzu die Gegenthese, wonach diese Hoffnungen kaum realistisch seien (vgl. Euler 2010). Zum einen würden Unternehmen nicht wesentlich ihre Ausbildungsanstrengungen ausbauen. Vor allem sei es unwahrscheinlich, dass sie aufgrund des nachlassenden Angebots an potentiellen Auszubildenden beginnen würden, verstärkt Jugendliche in die Ausbildung zu übernehmen, welche die allgemein bildenden Schulen mit kognitiven Lücken und/oder sozialen Schwierigkeiten verlassen. Vielmehr hätten Betriebe auch die Möglichkeit ihren Fachkräftebedarf alternativ, z. B. durch die Rekrutierung ausländischer Fachkräfte, zu decken (vgl. Euler 2010). Er und auch Severin und Hilger sehen vielmehr eine „MismatchProblematik" in der beruflichen Bildung heraufziehen mit einem Fachkräftemangel auf der einen und mit Bildungschancen unversorgten gering qualifizierten Jugendlichen auf der anderen Seite (vgl. Euler 2010; Hilger/Severing 2008).

Letztendlich legt der Vergleich der Argumentation der Verdrängungsthese und der Mismatch-Problematik die These nahe, dass nur dann eine Entlastung am Ausbildungsstellenmarkt eintreten kann, wenn das Ausbildungsstellenangebot langfristig nicht schneller sinkt als die Kohortenzahlen in den entsprechenden Altersjahrgängen. Außerdem wird deutlich, dass das Qualifikationsniveau der von Haupt- und Realschulen abgehenden Jugendlichen auch unter den Bedingungen des demografischen Wandels das zentrale Selektionskriterium darstellen wird.

Die Rolle des beruflichen Bildungssystems in Bezug auf den angenommenen baldigen Facharbeitermangel stellt das Thema vieler Veröffentlichungen und Fachtagungen dar. Beispielhaft zu nennen sind die BMBFKonferenz „Demografischer Wandel - Zukunft der beruflichen Aus- und Weiterbildung“ im Juni 2009, die KWB-Praxisbeiträge „Demographischer Wandel und Fachkräftebedarf: mit Berufsbildung Zukunft bewegen (vgl. KWB 2009) und die Veröffentlichung von Ulmer und Ulrich zum demografischen Wandel und seinen Folgen für die Sicherstellung des Fachkräftenachwuchses (vgl. Ulmer/Ulrich 2008). In Veröffentlichungen wird davon ausgegangen, dass die Bedeutung des Beitrags der Berufsbildung an der Sicherung 
des Fachkräftebedarfs steigen wird (vgl. Esser 2011: 3). Als „Basislager für den Fachkräftenachwuchs“ (Burgbacher 2010:1) sollen die Möglichkeiten des dualen Systems hierzu voll ausgeschöpft werden. Dabei wird kaum reflektiert, dass der demografische Wandel die berufsbildenden Schulen vor große Herausforderungen stellt, welche auch das Ziel einer optimalen Ausnutzung des Begabungspotentials durch berufliche Bildung beeinträchtigen dürfte (vgl. Maier/Troltsch/Walden 2011: 8).

\section{Demografischer Wandel und berufsbildende Schulen}

Wenn die Rolle der beruflichen Bildung vor dem Hintergrund des prognostizierten Fachkräftemangels beleuchtet wird, bleibt die Entwicklung der berufsbildenden Schulen in der Regel eigentümlicherweise unterbeleuchtet (vgl. KWB 2009; BmBF 2009). Dabei sind auch die berufsbildenden Schulen durch den demografischen Wandel zentral betroffen und die Möglichkeiten der Fachkräftesicherung sowie der Integration gering qualifizierter Jugendlicher durch berufliche Bildung wird nicht zuletzt auch davon abhängen, inwieweit es gelingt, Standorte berufsbildender Schulen in Zeiten schrumpfender Schülerjahrgänge zu sichern. Im folgenden werden Prognosen zur Entwicklung der Schülerzahlen in den berufsbildenden Schulen vorgestellt und am Beispiel von Nordrhein-Westfalen verdeutlicht, dass bereits jetzt strukturschwache Landkreise mit einem Schülerrückgang in den Berufsschulen konfrontiert sind.

Die Rahmenbedingungen berufsbildender Schulen sind dabei gekennzeichnet durch einen ansteigenden Anteil Jugendlicher mit Hochschulreife. So werden ab 2011 Studienberechtigungsquoten von über 50\% erwartet (vgl. Autorengruppe Bildungsberichterstattung 2010: 155). Außerdem sind sie geprägt von einem Rückgang der Schülerzahlen in allen Schulformen (vgl. Hetmeier/Schräbler/Schulz 2010). Dabei stellt sich dieser prognostizierte Rückgang höchst unterschiedlich dar, je nach dem welche Schulform der berufsbildenden Schule betrachtet wird und ob man auf die Flächenländer in Ost und West, oder aber auf die Stadtstaaten fokussiert.

Für die westdeutschen Flächenländer prognostizieren die statistischen Ämter des Bundes und der Länder bis zum Jahr 2024/25 einen Schülerrückgang von 27\% im dualen System, 30\% im Schulberufssystem und 35\% im Übergangssystem. Hier sollen die Schülerzahlen in den Schulformen, welche einen Hochschulzugang ermöglichen um 24\% zurückgehen (vgl. Hetmeier/Schräbler/Schulz 2010: 25).

In den Flächenländern Ost sollen die Schülerzahlen im Vergleich der Schuljahre 2008/09 und 2025/26 im dualen System um 27\% zurückgehen, im Schulberufssystem um 26\% und in den Schulformen, die zur Hochschulreife 
führen um 28\%. Im Übergangssystem liegt der Rückgang dagegen nur bei ca. 12\% (vgl. Hetmeier/Schräbler/Schulz 2010: 26).

Wieder anders stellt sich die Entwicklung in den Stadtstaaten dar, hier gehen die Schülerzahlen zunächst zurück, um im letzten Drittel des Prognosezeitraums wieder anzusteigen. Im Vergleich der Jahre 2008/09 und 2025/26 werden die Schülerzahlen im dualen System der Stadtstaaten um lediglich $5 \%$ und im Übergangssystem um 16\% abnehmen. Das Schulberufssystem und die Schulformen, die zur Hochschulreife führen, werden das Niveau des Jahres 2008/09 wieder erreichen (vgl. Hetmeier/Schräbler/Schulz 2010: 27). Bei rückläufigen Schülerzahlen in der Berufsschule als Teil des dualen Systems und dem Schulberufssystem kann davon ausgegangen werden, dass der Beitrag, den die berufliche Bildung zur Fachkräftesicherung leisten kann, ebenfalls rückläufig ist. Die rückläufigen Schülerzahlen dürften geringe Klassenstärken zur Folge haben, welche wiederum Schulstandorte gefährden könnten.

Betrachtet man als Beispiel der Entwicklung der Schülerzahlen in den berufsbildenden Schulen das Flächenland Nordrhein-Westfalen, so fällt auf, dass hier die Schülerzahlen im Zeitraum zwischen 1998 und 2008 stabil geblieben sind und sogar ein Anstieg von 18\% zu verzeichnen war. Allerdings legten die Schülerzahlen in den Teilzeitklassen, also im Berufsschulunterricht der dualen Ausbildung, lediglich um 8\% von 381.494 auf 410.369 Schülerinnen und Schüler zu. Der Hauptanteil des Zuwachses entfiel auf die Klassen der vollzeitschulischen Bildungsgänge, also auf Schulformen des Übergangssystems, vollzeitschulische Ausbildungsgänge und Schulformen, die zur Hochschulreife führen. Hier stieg die Schülerzahl von 136.921 auf 202.702 um $48 \%$ an (vgl. Schulministerium NRW 2010).

Tabelle 1: Entwicklung der Schülerzahlen an berufsbildenden Schulen in Nordrhein-Westfalen

\begin{tabular}{llll}
\hline & 1998 & 2008 & Veränd. \% \\
\hline Schulen & 362 & 358 & $-1 \%$ \\
SchülerInnen & 518.406 & 613.070 & $18 \%$ \\
Vollzeit & 136.921 & 202.702 & $48 \%$ \\
Teilzeit & 381.494 & 410.368 & $8 \%$ \\
\hline
\end{tabular}

Quelle: Schulministerium NRW 2010/eigene Berechnungen

Im Zuge des Projekts „Wegweiser Kommune“ weist die BertelsmannStiftung regional differenzierte Prognosen zur Bevölkerungsentwicklung aus. In Nordrhein-Westfalen sind neun Kreise besonders stark von einem zukünftige Bevölkerungsrückgang betroffen, d. h. hier wird im Zeitraum von 2006 bis 2025 mit einem Rückgang von 6 bis 10\% der Bevölkerung gerechnet 
(vgl. Bertelsmannstiftung 2010). In vier dieser neun Kreise gingen bereits im Zeitraum von 1998 bis 2008 die Schülerzahlen in den Teilzeitklassen zurück, was aber, bezogen auf die Gesamtschülerzahl durch das starke Anwachsen der Schülerzahlen in den vollschulischen Klassen aufgefangen werden konnte.

Tabelle 2.: Entwicklung der Schülerzahlen in vom demografischen Wandel stark betroffenen Landkreisen Nordrhein-Westfalens

\begin{tabular}{|l|l|l|l|l|l|}
\hline \multirow{2}{*}{ Kreis } & \multicolumn{5}{|c|}{ Entw. Schülerzahlen 1998- } \\
\cline { 2 - 6 } En.-Ruhr & $\mathrm{TZ}$ & $4 \%$ & Mettman & $\mathrm{TZ}$ & $9 \%$ \\
& $\mathrm{VZ}$ & $61 \%$ & & $\mathrm{VZ}$ & $59 \%$ \\
Hochsauerland & $\mathrm{TZ}$ & $-\boldsymbol{8} \%$ & Rhein Sieg & $\mathrm{TZ}$ & $6 \%$ \\
Lipper & $\mathrm{VZ}$ & $55 \%$ & & $\mathrm{VZ}$ & $80 \%$ \\
& $\mathrm{TZ}$ & $-13 \%$ & Siegen Wittgenstein & $\mathrm{TZ}$ & $11 \%$ \\
Märkischer Kreis & $\mathrm{VZ}$ & $27 \%$ & & $\mathrm{VZ}$ & $57 \%$ \\
& $\mathrm{TZ}$ & $-1 \%$ & Recklinghausen & $\mathrm{TZ}$ & $-2 \%$ \\
& $\mathrm{VZ}$ & $53 \%$ & & $\mathrm{VZ}$ & $60 \%$ \\
& $\mathrm{TZ}$ & $6 \%$ & & & \\
\hline
\end{tabular}

TZ: Teilzeitklassen; VZ: Vollzeitklassen

Quelle: Schulministerium NRW 2010/eigene Berechnungen

Diese Zahlen deuten auf die Gefahr einer durch den demografischen Wandel induzierte Abwärtsspirale für das duale System im ländlichen Raum hin. Wenn wegen der rückläufigen Kohortenstärken in den für die berufliche Bildung zentralen Altersklassen nur noch geringe Klassenstärken erreichbar sind, kann das zu Schließungen und Zusammenlegungen von Schulstandorten führen.

Solche Schließungen und Zusammenlegungen könnten aber gerade im ländlichen Raum zur Folge haben, dass die Attraktivität des dualen Systems sinkt, da die Distanzen zwischen den Lernorten Betrieb und Berufsschule steigt. Zusätzlich könnte auch der Rückbau des Übergangssystems die beruflichen Schulen in Existenznöte bringen, da dieses über die vollschulischen Schulformen in den letzten Jahren zunehmen mehr Unterrichtskapazitäten gebunden hatte und es auch in Zeiten schwacher Entwicklung der Schülerzahlen in den Berufsschulen des dualen Systems einen Ausgleich darstellte. 


\section{Ansätze zur Entwicklung berufsbildender Schulen}

Die Situation, dass auf der einen Seite berufliche Bildung aktiv an der Sicherung des Fachkräfteangebotes mitwirken soll und gleichzeitig selbst massiv durch den demografischen Wandel betroffen ist, wirft die Frage auf, welche Ansätze zur strukturellen Entwicklung berufsbildender Schulen bestehen, die das Problem des demografischen Wandels adressieren.

Aktuell wird seitens einiger Landesregierungen versucht, strukturell den Herausforderungen der berufsbildenden Schulen zu begegnen. Obschon viele dieser Versuche in erster Linie darauf abzielen, eine Antwort auf die Übergangsproblematik der Jugendlichen ohne Ausbildungsplatz und auf die Vielfalt der Bildungsangebote an berufsbildenden Schulen zu geben, ermöglichen sie auch eine Anpassung an die Veränderungstendenzen aufgrund des demografischen Wandels.

Das in Hessen ins Leben gerufene Programm Hessencampus stellt z. B. den Versuch dar, auf regionaler Ebene Zentren Lebenslangen Lernens zu schaffen, um in den Regionen die Bildungsbeteiligung zu erhöhen, lebenslanges Lernen zu fördern und Übergänge in Beschäftigung zu unterstützen (vgl. Kruse et. al. 2009). Dies soll durch eine regionale Kooperation unterschiedlicher Bildungsträger wie berufsbildende Schulen, Abendgymnasien und Volkshochschulen erreicht werden, die auf die Schaffung von Synergien und eine gebündelte Ressourcennutzung abstellt. Solche Kooperationen können natürlich auch dazu dienen, unter den Voraussetzungen rückläufiger Schülerzahlen Bildungsangebote in der Region zu sichern. So werden z. B. in der Region Odenwald in Folge des Hessencampus-Projekts berufsbildende Schulen, ein Abendgymnasium und eine Außenstelle der Volkshochschule gebündelt, um Bildungsangebote auch unter den Auswirkungen des demografischen Wandels aufrechterhalten zu können (vgl. BSO 2011).

Zur Flexibilisierung von Schulformen und Ausbildungsgängen wurde in Rheinland-Pfalz in den berufsbildenden Schulen eine Curriculumskonzeption implementiert, welche unter anderem abschlussbezogene Lernbausteine in den berufsübergreifenden Fächern an den berufsbildenden Schulen vorsieht. Hierdurch erhofft man sich die Möglichkeit, zielgruppenspezifisch passgenaue Angebote in den berufsbildenden Schulen realisieren zu können und einen Schritt in Richtung Gleichwertigkeit von allgemeiner und beruflicher Bildung zu verwirklichen. Synergieeffekte sollen durch die Anerkennung von Vorleistungen ermöglicht werden. Darüber hinaus entstehen aber auch Synergieeffekte dadurch, dass in den berufsübergreifenden Fächern Schülerinnen und Schüler unterschiedlicher Schulformen an den berufsbildenden Schulen bei rückläufigen Jahrgangsstärken flexibel zusammengefasst werden können (vgl. Pädagogisches Zentrum o. J.). 
Eine vom Bundesministerium für Bildung und Forschung veröffentlichte Studie zu den ,Auswirkungen von demographischen Entwicklungen auf die berufliche Ausbildung“" (Pfeifer/Kaiser 2009) spricht konkrete Empfehlungen zur Strukturentwicklung berufsbildender Schulen aus. Demnach sollen neben Bundes- und Fachklassen virtuelle Bildungsangebote in der beruflichen Bildung ausgebaut werden. Als Beispiel sollen Aufbau und Gestaltung von Fernstudiengängen an Hochschulen dienen. Auch ist von Internatsplätzen für Jugendliche an berufsbildende Schulen unter den Vorzeichen eines ,gesteuerten Rückbaus“ die Rede (vgl. Pfeifer/Kaiser 2009: 80). Maßnahmen zur Steigerung der Mobilität der Jugendlichen und ein flächendeckender Aufbau branchenspezifischer regionaler Aus- und Weiterbildungszentren werden propagiert (vgl. Pfeifer/Kaiser 2009: 75 f.).

Insgesamt kann resümiert werden, dass der offenen Frage, wie in Zeiten des demografischen Wandels die Versorgung mit Berufsschulstandorten für das duale System gesichert werden kann bisher mit vage Perspektiven begegnet wird, die sich an Konzepte einer verstärkten Kooperation von Bildungsinstitutionen und eine regional gesteuerte Nutzung der Bildungsinstitutionen im Prozess des lebenslangen Lernens anlehnen (vgl. Kruse et. al. 2009). In den ostdeutschen Flächenländern hat der demografische Wandel bereits zu einem massiven Rückbau an berufsbildenden Schulen geführt, der Probleme der Aufrechterhaltung des beruflichen Bildungsangebotes in einwohnerschwachen Landkreisen zur Folge hat. Hier wird bereits von einer Entwicklung von regionalen und Bezirksfachklassen hin zu Landes- und länderübergreifenden Fachklassen gesprochen (vgl. Kuklinski 2006).

\section{Anpassungsproblemen und Forschungsfragen}

Erstaunlich ist angesichts der problematischen Entwicklungstendenzen der berufsbildenden Schulen, dass, wie eingangs beschrieben, der beruflichen Bildung eine herausgehobene Rolle bei der Bereitstellung von Fachkräften in Zeiten des demografischen Wandels zukommen soll, aber auf die Probleme am Lernort „berufsbildenden Schule“ kaum eingegangen wird. Das Problem der Aufrechterhaltung berufsbildenden Unterrichts in vom demografischen Wandel besonders betroffenen Regionen wird in diesem Kontext nicht nur kaum diskutiert, es fehlen generell berufspädagogisch fundierte Strategien der Schulentwicklung, die dezidiert auf die Problematik des demografischen Wandels eingehen. Die naheliegende Frage, wie berufsbildende Schulen unter den veränderten Rahmenbedingungen ihren Beitrag zur Sicherung des Fachkräftebedarfs leisten sollen, muss zunächst unvollständig beantwortet bleiben. 
Als Forschungsfrage steht im Raum, wie ein demografiebedingter Rückbau berufsbildender Schulen gelingen kann, ohne dass die Attraktivität beruflicher Bildung zurückgeht und auch in strukturschwachen Landkreisen die berufliche Bildung ihre Attraktivität behalten kann, um die Begabtenreserven vor dem Hintergrund des Fachkräftemangels optimal auszuschöpfen. Dabei sind berufsbildende Schulen seit geraumer Zeit mit dem Problem schwankender Schülerzahlen in den unterschiedlichen Schulformen konfrontiert und erproben traditionell eine Vielzahl von curricularen und unterrichtsorganisatorischen Modellen, um dieser Herausforderung zu begegnen. Eine Untersuchung und Evaluation dieser Modelle unter dem Hinblick auf die Tauglichkeit in Bezug auf die Anforderungen des demografischen Wandels wäre eine lohnende Forschungsaufgabe. Auch wäre es lohnend, die Auswirkungen des demografischen Wandels auf die Systementwicklung beruflicher Bildung in den neuen Bundesländern zu untersuchen, da diese bereits seit geraumer Zeit mit dem Problem konfrontiert sind. Hier stellen sich die Forschungsfragen, wie ein Berufsbildungssystem wie das ostdeutsche, das viel stärker durch staatlich geförderte Maßnahmen und weniger durch das duale System gekennzeichnet ist, auf den demografischen Wandel reagiert.

Letztendlich hängen auch mit dem demografischen Wandel verbundene Hoffnungen über eine verstärkte Integration sozialer Gruppen, die aktuell am Ausbildungsstellenmarkt benachteiligt sind (Jugendliche mit Migrationshintergrund, Jugendliche mit Hauptschulabschluss) davon ab, wie die berufsbildenden Schulen im Hinblick auf die Herausforderung des demografischen Wandels aufgestellt sind.

\section{Literatur}

Autorengruppe Bildungsberichterstattung (2010): Bildung in Deutschland 2010. Ein indikatorengestützter Bericht mit einer Analyse zu Perspektiven des Bildungswesens im demographischen Wandel. Bielefeld.

Bertelsmann Stiftung (2010): Länderbericht Nordrhein-Westfalen. Online: www. wegweiser-kommune.de/datenprognosen/laenderberichte/download/pdf/ Laender bericht_Nordrhein_Westfalen.pdf. 12.01.2011.

BmBF (2009): Auswirkungen von demographischen Entwicklungen auf die berufliche Ausbildung. Bonn/Berlin.

BSO - Berufliches Schulzentrum Odenwaldkreis (2010): Der Hessencampus Odenwaldkreis füllt sich. Online: www.bso-michelstadt.de/index.php?id=257\&L=2. 05.01.2011. 
Burgbacher, E. (2010): Thesenpapier zu der Rede: „Berufliche Bildung als Basislager für Fachkräftenachwuchs und Innovationstreiber der Wirtschaft" anlässlich des 56. IW-Studiengesprächs zur beruflichen Bildung „Berufsausbildung im Zeichen der Wirtschaftskrise“. Online: http://www.iwkoeln.de/Portals/0/pdf/ veranstaltungen/2010/020510_burgbacher.pdf. 13.07.2011.

Castells, M. (2004): Der Aufstieg der Netzwerkgesellschaft. Bd. 1: Das Informationszeitalter. Opladen.

Euler, D. (2010): Einfluss der demographischen Entwicklung auf das Übergangssystem und den Berufsausbildungsmarkt. Online: www.bertelsmann-stiftung. de/cps/ rde/xbcr/SID-6D015A25-7DB7C1F3/bst/xcms_bst_dms_32527_32528_2.pdf. 12.01. 2011.

Esser, F. H. (2011): Fachkräftesicherung ist originäre Aufgabe der Berufsbildung. In: Berufsbildung in Wissenschaft und Praxis, 3, S. 3.

Hetmeier, H.-W./ Schräpler, J.-P./ Schulz, A. (2010): Vorausberechnung der Bildungsteilnehmerinnen und Bildungsteilnehmer, des Personal- und Finanzbedarfs bis 2025. Online: www.destatis.de/jetspeed/portal/cms/Sites/destatis/Internet/ DE/Content/Publikationen/Fachveroeffentlichungen/BildungForschungKultur/Bil dungsvorausberchnung,property=file.pdf. 25.01.2011.

Hilger, A./ Severing, E. (2008): Berufsausbildung in Deutschland - Zu wenige Fachkräfte für die Wirtschaft und zu viele Jugendliche im Übergangssystem. In: Münk, D./ Rützel, J./ Schmidt, C.: Labyrinth Übergangssystem. Forschungserträge und Entwicklungsperspektiven der Benachteiligtenförderung zwischen Schule Ausbildung, Arbeit und Beruf. S. 93-103.

Kruse, W./ Kaletka, C./ Pelka, B./ Schröder, A. (Hrsg.)(2009): Hessencampus 20072009. Veröffentlichungen der wissenschaftlichen Begleitung. Dortmund.

Kuklinski, P. (2006): Demografischer Wandel und Schulnetzplanung für berufsbildende Schulen - dargestellt am Beispiel der Situation in Sachsen. In: Recht der Jugend und des Bildungswesens, 4, S. 474-493.

KWB - Kuratorium der Deutschten Wirtschaft für Berufsbildung (Hrsg.) (2007): Herausforderung Demographischer Wandel. Berufliche Bildung in Verantwortung für die Zukunft. Bonn.

Pädagogisches Zentrum (o. J.): Berufsbildende Schule Rheinland-Pfalz. Strukturkonzept. Allgemeine Informationen. Online: http://bbs.bildung-rp.de/no-cache/ materialien.html?tx_abdownloads_pi $1 \% 5$ Baction $\% 5 \mathrm{D}=$ getviewclickeddownload\&tx _abdownloads_pi1\%5Buid $\% \overline{5 D}=117.05 .06 .2010$.

Prognos AG (2010): Arbeitslandschaft 2030 - Auswirkungen der Wirtschafts- und Finanzkrise.

Schulministerium NRW (2010): SchulInfo NRW. Online: http://www. schulministerium.nrw.de/BP/Schulsystem/Statistik. 25.01.2011.

Solga, H. (2005): Ohne Abschluss in die Bildungsgesellschaft. Die Erwerbschancen gering qualifizierter Personen aus ökonomischer und soziologischer Perspektive. Opladen.

Ulmer, P./ Ulrich, J. G. (Hrsg.) (2009): Der demografische Wandel und seine Folgen für die Sicherstellung des Fachkräftenachwuchses. Wissenschaftliche Diskussionspapiere, Heft 206. Online: www.bibb.de/dokumente/pdf/wd_106_demografischer_wandel_und_seine_folgen.pdf. 04.04.2011. 